\title{
A Simple Approach to the Perron-Frobenius Theory for Positive Operators on General Partially-Ordered Finite-Dimensional Linear Spaces*
}

\author{
By Werner C. Rheinboldt and James S. Vandergraft
}

\begin{abstract}
This paper presents simple proofs of the principal results of the Perron-Frobenius theory for linear mappings on finite-dimensional spaces which are nonnegative relative to a general partial ordering on the space. The principal tool for these proofs is an application of the theory of norms in finite dimensions to the study of order inequalities of the form $A x \leqq \alpha x, x \geqq 0$ where $A \geqq 0$. This approach also permits the derivation of various inclusion and comparison theorems.
\end{abstract}

1. Introduction. The results of Perron [1907] and Frobenius [1908]-[1912] concerning spectral properties of matrices with nonnegative elements have become an important tool in the study of iterative methods for linear equations in $R^{n}$. These results have been generalized in various ways; see, for example, Krein and Rutman [1950] and Schaefer [1966] for general extensions to infinite-dimensional spaces and further references.

Simple proofs of the Perron-Frobenius results for matrices can be found in Varga [1962] and Householder [1964]. These proofs, however, do not appear to carry over to the case of linear mappings on a finite-dimensional space which are nonnegative under a general partial ordering on the space. For this case, it is necessary either to emulate the infinite-dimensional proofs by using the Brouwer fixed point theorem (see, e.g., Fan [1958]) or to depend heavily on the spectral theory of finite-dimensional linear maps and the Jordan form of a matrix (see Birkhoff [1967] and Vandergraft [1968]).

In this paper, elementary proofs are presented of the principal results of the Perron-Frobenius theory for general partially-ordered finite-dimensional spaces. Our basic tools are some results about norms and a consistent use of simple orderbound concepts. No use is made of the spectral theory of linear mappings. These proofs are similar in spirit to the cited proofs of Varga and Householder for the case of the componentwise ordering. They also emulate some techniques of Bohl [1966] and Schneider and Turner [1972] which were employed by these authors in connection with discussions of the infinite-dimensional case.

2. Preliminary Results. Let $V$ be a finite-dimensional, normed, real, linear space, and $K \subset V$ a closed, convex, and solid cone. In other words, $\alpha K \subset K$ for all $\alpha \geqq 0, K+K \subset K, K \cap(-K)=\{0\}$, and the interior $K^{0}$ of $K$ is nonempty. These

Received April 24, 1972.

AMS (MOS) subject classifications (1970). Primary 15A48; Secondary 65F10.

* This research was sponsored in part by Grant GJ-1067 from the National Science Foundation to the Computer Science Center of the University of Maryland. 
assumptions ensure that the cone is generating, that is, $V=K-K$. The partial ordering induced by $K$ is denoted by $\geqq$. Thus, $x \geqq y$ means $x-y \in K$, and $x>y$ stands for $x-y \in K^{0}$. If $A \in L(V)$ is a linear mapping of $V$ into itself, then $A \geqq 0$ is defined by $A x \in K$ whenever $x \in K$.

If $u \in K^{0}$, then the order interval

$$
[-u, u]=\{x \in V \mid-u \leqq x \leqq u\}
$$

is closed and convex. Moreover, for any $x \in V$, there exists a $t>0$ such that $x \in$ $t[-u, u]$ (absorption property), and, if $x \in[-u, u]$, then $\alpha x \in[-u, u]$ for any $\alpha$ with $|\alpha| \leqq 1$ (equilibration property). Thus, a norm on $V$ is defined by

$$
\|x\|_{u}=\inf \{t \geqq 0 \mid x \in t[-u, u]\}
$$

(see Householder [1964, p. 38]). This norm is monotonic; that is, $0 \leqq x \leqq y$ implies that $\|x\|_{u} \leqq\|y\|_{u}$ and the induced operator norm

$$
\|A\|_{u}=\sup _{\|x\|_{u}=1}\|A x\|_{u}, \quad A \in L(V)
$$

satisfies

$$
\|A\|_{u}=\|A u\|_{u}, \quad A \in L(V), A \geqq 0 .
$$

Next, for any norm on $L(V)$ induced by a norm on $V$, we write

$$
r(A)=\underset{k \rightarrow \infty}{\limsup }\left\|A^{k}\right\|^{1 / k} .
$$

Since all norms on $V$ are equivalent, $r(A)$ is norm-independent. Also, since $\left\|A^{k}\right\| \leqq$ $\|A\|^{k}$, it follows that $r(A) \leqq\|A\|$ under any norm. We shall not use the well-known fact that the lim sup is actually a limit, nor that $r(A)$ is the spectral radius of $A$. The facts about $r(A)$ which we do need are contained in the following lemma.

LEMmA 1. If $A \in L(V)$, then the following statements are equivalent.

(i) $r(A)<1$,

(ii) $\sum_{k=0}^{\infty} A^{k}$ converges uniformly to $(I-A)^{-1}$,

(iii) $\lim _{k \rightarrow \infty} A^{k}=0$.

Proof. (i) $\Rightarrow$ (ii) From $r(A)=\lim \sup \left\|A^{k}\right\|^{1 / k}<1$ follows the convergence of $\sum_{k=0}^{\infty}\left\|A^{k}\right\|$ which is exactly the statement that $\sum A^{k}$ converges uniformly. The fact that the series represents $(I-A)^{-1}$ is a consequence of

$$
\left(I+A+A^{2}+\cdots+A^{m}\right)(I-A)=I-A^{m+1} \text {. }
$$

(ii) $\Rightarrow$ (iii) If $\sum\left\|A^{k}\right\|$ converges, then necessarily $\lim _{k \rightarrow \infty}\left\|A^{k}\right\|=0$.

(iii) $\Rightarrow$ (i) Let $\alpha_{k}=\left\|A^{k}\right\|$. Then the following facts are obvious:

(a) $\alpha_{k} \geqq 0, \forall k \geqq 0$,

(b) $\alpha_{a}<1$, for some $q>0$,

(c) $\alpha_{k+j} \leqq \alpha_{k} \alpha_{i}, \forall k, j \geqq 0$.

Without loss of generality, we may assume that $\alpha_{q}>0$. Now, any integer $m>0$ can be written as $m=p q+r$, where $p \geqq 0,0 \leqq r<q$. Thus,

$$
\alpha_{m}=\alpha_{p q+r} \leqq\left(\alpha_{q}\right)^{p} \alpha_{r}=\alpha_{q}^{m / q} \alpha_{q}^{-r / q} \alpha_{r}=\left(\alpha_{q}^{1 / q}\right)^{m} \beta_{r}
$$

where $\beta_{r}=\alpha_{r} \alpha_{q}^{-r / q}$. Hence, $\alpha_{m}^{1 / m} \leqq \alpha_{q}^{1 / q} \beta_{r}^{1 / m}$ and, since there are only finitely many $\beta_{r}$, it follows that $\lim _{m \rightarrow \infty} \beta_{r}^{1 / m}=1$; that is 


$$
r(A)=\limsup _{m \rightarrow \infty} \alpha_{m}^{1 / m} \leqq \alpha_{q}^{1 / a}<1
$$

3. Order Bounds. The theorems in this section are based on order inequalities of the form $A x \leqq \alpha x$, for some $x \geqq 0$. Such inequalities play a considerable role in most of the works cited in the introduction; however, their full value in the finitedimensional case does not seem to have been realized. We begin with a convergence result.

TheOREM 2. Let $A \geqq 0, A \in L(V)$, and suppose that $A x \leqq x$ for some $x>0$. Then $\lim _{k \rightarrow \infty} A^{k} x=x^{*} \leqq x$ exists. Moreover, $x^{*}=0$ if and only if $r(A)<1$.

Proof. Set $x^{k}=A^{k} x, k \geqq 0$; then, by induction,

$$
0 \leqq x^{k+1} \leqq x^{k} \leqq \cdots \leqq x^{1} \leqq x
$$

and hence $\lim x^{k}=x^{*}$ exists, and $x^{*} \leqq x$. If $r(A)<1$, then Lemma 1 implies that $\lim _{k \rightarrow \infty} A^{k}=0$, and hence that $\lim _{k \rightarrow \infty} A^{k} x=0$. If $x^{*}=0$, then

$$
\left\|A^{k}\right\|_{x}=\left\|A^{k} x\right\|_{x} \rightarrow\left\|x^{*}\right\|_{x}=0, \quad \text { as } k \rightarrow \infty,
$$

and, thus, $\lim _{k \rightarrow \infty} A^{k}=0$, which by Lemma 1 implies that $r(A)<1$.

The first part of this theorem is a linear version of a lemma of Kantorovich [1939] which has been used extensively in the study of iterative methods for nonlinear equations. In order to apply this result, it is necessary to find $x>0$ with $A x \leqq x$. The next theorem provides such an $x$.

TheOREM 3. Let $A \geqq 0, A \in L(V)$. Then $A x<\alpha x$ for some $x>0$, and $\alpha>0$, if and only if $\alpha>r(A)$.

Proof. Suppose that $\alpha>r(A)$. Then $\hat{A}=(1 / \alpha) A$ satisfies $r(\hat{A})<1$, and, by Lemma 1 , we have

$$
(I-\hat{A})^{-1}=\sum_{k=0}^{\infty} \hat{A}^{k}
$$

and, clearly, $(I-\hat{A})^{-1} \geqq 0$ since all terms in the series are nonnegative. If $y>0$, then $x=(I-\hat{A})^{-1} y$ satisfies $(I-\hat{A}) x>0$, whence $0 \leqq A x<\alpha x$ and $x>0$. Conversely, if $A x<\alpha x$ for some $x>0$, then $A x \leqq(\alpha-\epsilon) x$ for some $\epsilon>0$, and hence

$$
r(A) \leqq\|A\|_{x}=\|A x\|_{x} \leqq(\alpha-\epsilon)<\alpha .
$$

The special case $\alpha=1$ of this theorem was proved by Vandergraft [1972] using the Perron-Frobenius theory in partially-ordered spaces. In that paper, a matrix $B$ such that $B x>0$ for some $x>0$ is called $K$-semipositive. Hence, Theorem 3 states that, for $A \geqq 0, \alpha I-A$ is $K$-semipositive if and only if $\alpha>r(A)$.

The next theorem concerns the case when some strict inequalities in the previous theorem are reduced to simple ones.

THEOREM 4. Let $A \geqq 0, A \in L(V)$. If $r(A) \leqq \alpha$, then there exists an $x \geqq 0$, $x \neq 0$ with $A x \leqq \alpha x$. Conversely, if $A x \leqq \alpha x$ for some $x>0$, then $r(A) \leqq \alpha$; and if $r(A)=\alpha>0$, then $A x^{*}=r(A) x^{*}$ for some $0 \leqq x^{*} \leqq x, x^{*} \neq 0$.

Proof. If $r(A) \leqq \alpha$, then $r(A)<t_{k}=\alpha+1 / k, k=1,2, \cdots$. By Theorem 3, there exist $x^{k}>0, k=1,2, \cdots$, with $A x^{k} \leqq t_{k} x^{k}$. These vectors $x^{k}$ can be scaled so that $\left\|x^{k}\right\|=1$ for all $k$, which implies that a convergent subsequence can be found with a limit $x^{*}$ satisfying $A x^{*} \leqq \alpha x^{*}, x^{*} \geqq 0,\left\|x^{*}\right\|=1$. 
For the converse, we have $r(A) \leqq\|A\|_{x}=\|A x\|_{x} \leqq \alpha$; and, if $r(A)=\alpha>0$, then $\hat{A}=(1 / \alpha) A$ satisfies $r(\hat{A})=1$. Now, apply Theorem 2 to $\hat{A}$ to find $x^{*} \neq 0$ with $0 \leqq x^{*} \leqq x$ and $\hat{A} x^{*}=x^{*}$, that is, $A x^{*}=\alpha x^{*}=r(A) x^{*}$.

4. $K$-Irreducibility. Theorem 4 establishes the existence of a vector $x \geqq 0$ such that $A x \leqq r(A) x$. In order to prove that equality holds for some nontrivial $x \geqq 0$, it is convenient to impose slightly stronger conditions on $A$. In the case of the natural (componentwise) ordering on $R^{n}$, the concept of irreducibility is used in this connection. Vandergraft [1968] extended this concept to general partial orderings by considering the face structure of the cone $K$. For our purposes, it is expedient to use a somewhat simpler but equivalent definition.

Definition 5. The mapping $A \in L(V), A \geqq 0$, is $K$-irreducible if $A x \leqq \alpha x$ for some $x \geqq 0, x \neq 0$, implies that $x>0$. Otherwise, it is called $K$-reducible.

Note that by this definition $A \geqq 0$ is $K$-reducible if $A x \leqq \alpha x$ for some $x \neq 0$ on the boundary $\partial K$ of $K$.

The equivalence of this definition with that given by Vandergraft [1968] follows from Lemma 2.1 of that paper. In fact, if $A x \leqq \alpha x$, for $x \neq 0, x \in \partial K$, then this lemma ensures the existence of a linear subspace $S \subset V$, such that $K \cap S$ is a subset of $\delta K$, and $A$ restricted to $S$ maps $K \cap S$ into itself. This is equivalent to Vandergraft's earlier definition of $K$-reducibility.

Theorem 6. If $A \in L(V), A \geqq 0$, is $K$-irreducible, then $A x^{*}=r(A) x^{*}$ for some $x^{*}>0$ and $r(A)>0$.

Proof. By Theorem 4, we know that $A x \leqq r(A) x$ for some $x \geqq 0, x \neq 0$, and by $K$-irreducibility it follows that $x>0$. Now, $r(A) \geqq\|A x\|_{x}=\|A\|_{x}>0$, so Theorem 4 also ensures that $A x^{*}=r(A) x^{*}$ for some $0 \leqq x^{*} \leqq x, x^{*} \neq 0$, which by $K$-irreducibility is only possible for $x^{*}>0$.

In order to prove a similar theorem for $K$-reducible $A \geqq 0$, we use a standard continuity argument. Note first that there are $B \in L(V)$ such that $B x>0$ for any $x \geqq 0, x \neq 0$. In fact, if $u>0$ and $S \oplus \operatorname{span}(u)=V$ is any decomposition of $V$, then the induced projection $B$ from $V$ onto $\operatorname{span}(u)$ has this property. Now, let $A \in$ $L(V), A \geqq 0$ be given, and let $\epsilon>0$. Then, by Theorem $3, A u<(r(A)+\epsilon) u$ for some $u>0$, and thus,

$$
r(A) \leqq\|A\|_{u}=\|A u\|_{u} \leqq r(A)+\epsilon .
$$

Furthermore, the maps $A_{k}=A+(1 / k) B, k \geqq 1$, are $K$-irreducible and, hence, we have $A_{k} x^{k}=r\left(A_{k}\right) x^{k}$, where $x^{k}>0$, and we may assume that $\left\|x^{k}\right\|_{u}=1$, for all $k$. Since $A x^{k} \leqq r\left(A_{k}\right) x^{k}, x^{k}>0$, we have by Theorem 4 and (1), that

$$
r(A) \leqq r\left(A_{k}\right) \leqq\left\|A_{k}\right\|_{u} \leqq\|A\|_{u}+\frac{1}{k}\|B\|_{u} \leqq r(A)+\epsilon+\frac{1}{k}\|B\|_{u} .
$$

Let $\left\{x^{k_{i}}\right\}$ be a convergent subsequence, with limit $x^{*} \geqq 0, x^{*} \neq 0$. Then, from (2), it follows that $\lim _{k \rightarrow \infty} r\left(A_{k}\right)=r(A)$, and thus $A x^{*}=r(A) x^{*}$. This proves the following result.

THEOREM 7. For any $A \geqq 0, A \in L(V)$ with $r(A)>0$, there exists an $x^{*} \geqq 0$, $x^{*} \neq 0$, such that $A x^{*}=r(A) x^{*}$.

$K$-irreducibility also allows a strengthening of some earlier results. The next theorem, for example, is a stronger form of the second part of Theorem 4. 
THEOREM 8. Let $A \geqq 0$ be $K$-irreducible, and suppose that $A x \leqq \alpha x, A x \neq \alpha x$ where $x>0, \alpha>0$. Then $r(A)<\alpha$.

Proof. By Theorem 4, $r(A) \leqq \alpha$. If $r(A)=0$, then $r(A)<\alpha$; hence suppose that $r(A)=\alpha \neq 0$. By Theorem 6, $A x^{*}=\alpha x^{*}$ where $x^{*}>0$. Now, if $z=\left\|x^{*}\right\|_{x} x-$ $x^{*}$, then $z \in \delta K$ and

$$
0 \leqq A z=\left\|x^{*}\right\|_{x} A x-A x^{*} \leqq\left\|x^{*}\right\|_{x} \alpha x-\alpha x^{*}=\alpha z,
$$

which contradicts the $K$-irreducibility of $A$.

As an application of this result, we give a simple proof of the monotonicity of $r(A)$.

Theorem 9. Let $0 \leqq A \leqq B$ where $A$ is $K$-irreducible and $A \neq B$. Then $r(A)<$ $r(B)$.

Proof. Clearly, $B$ is also $K$-irreducible, and by Theorem $6, r(B)>0, B x^{*}=$ $r(B) x^{*}$ with $x^{*}>0$. But then $A x^{*} \leqq B x^{*}=r(B) x^{*}$ and $A x^{*} \neq r(B) x^{*}$, which, by Theorem 8, gives $r(A)<r(B)$.

The remaining statements of the Perron-Frobenius theory can be proven in the standard way (see, e.g., Vandergraft [1968] and Householder [1964]). We shall not go into detail here, but rather consider results like those of Section 3 with certain inequalities reversed. An example of such a theorem is as follows.

Theorem 10. Let $A \geqq 0, A \in L(V)$ be $K$-irreducible. If $A x \geqq \alpha x, A x \neq \alpha x$ for some $x \geqq 0, \alpha>0$, then $r(A)>\alpha$. Conversely, if $r(A)>\alpha$, then $A x>\alpha x$ for some $x>0$.

Proof. If $A x \geqq \alpha x, A x \neq \alpha x, x \geqq 0, \alpha>0$, then $\hat{A} x \geqq x$, where $\hat{A}=(1 / \alpha) A$, and, by Theorem $6, \hat{A} x^{*}=r(\hat{A}) x^{*}, x^{*}>0$. Now $\|x\|_{x^{*}} x^{*}-x \geqq 0$, and hence

$$
0 \leqq \hat{A}\left(\|x\|_{x^{*}} x^{*}-x\right) \leqq\|x\|_{x^{*}} r(\hat{A}) x^{*}-x
$$

or $x \leqq r(\hat{A})\|x\|_{x^{*}} x^{*}$ which, by definition of $\|x\|_{x^{*}}$ implies that $r(\hat{A}) \leqq 1$. But $r(\hat{A})=1$ and (3) contradict the $K$-irreducibility of $A$; hence $r(\hat{A})<1$; that is, $r(A)<\alpha$. Conversely, by Theorem $6, A x^{*}=r(A) x^{*}$, with $x^{*}>0$, and thus $r(A)>\alpha$ implies that $A x^{*}=r(A) x^{*}>\alpha x^{*}$.

Using the usual continuity argument, we can drop the $K$-irreducibility in Theorem 9, and this leads to the following theorem.

THEOREM 11. Let $A \geqq 0, A \in L(V)$. Then $A x \geqq \alpha x$ for some $x \geqq 0, x \neq 0$, $\alpha>0$, if and only if $r(A) \geqq \alpha$.

Upper and lower bounds for $r(A)$ can be obtained by combining Theorems 8 and 10 for $K$-irreducible matrices, or Theorems 4 and 11 in the $K$-reducible case. We state the result only for the $K$-irreducible case.

THEOREM 12. If $A \geqq 0, A \in L(V)$, is $K$-irreducible, then $\alpha x \leqq A x \leqq \beta x$, with equality excluded, for some $x \geqq 0, x \neq 0$, implies that $\alpha<r(A)<\beta$.

This provides a simple proof of a well-known inclusion theorem of Frobenius [1908]. That is, if $A$ is an irreducible matrix in the classical sense, then applying Theorem 12 to $|A|=\left(\left|a_{i j}\right|\right)$, with the vector $x=(1,1, \cdots, 1)^{T}$ and the usual componentwise ordering, we find that $s<r(A)<S$, where

$$
s=\min _{i} \sum_{i=1}^{n}\left|a_{i i}\right|, \quad S=\max _{i} \sum_{i=1}^{n}\left|a_{i j}\right| .
$$

Theorem 4 can also be used to prove the following well-known result. 
Theorem 13. If $A \geqq 0, A \in L(V)$, then $A x=\alpha x, x>0$, implies that $\alpha=r(A)$.

Proof. By Theorem 4, we have $r(A) \leqq \alpha$. If $\alpha>r(A)$, then $\alpha \neq 0$, and $\hat{A}=$ $(1 / \alpha) A$ satisfies $\hat{A} x=x$. Hence, $\hat{A}^{k} x=x$ and $\left\|\hat{A}^{k}\right\|_{x}=\left\|\hat{A}^{k} x\right\|_{x}=\|x\|_{x}=1$, for all $k \geqq 0$, which means that $\left\{\hat{A}^{k}\right\}$ does not converge to zero. Lemma 1 then states that $r(\hat{A}) \geqq 1$; that is, $r(A) \geqq \alpha$ and, altogether, $r(A)=\alpha$.

As a final application, we give a simple proof of the Stein-Rosenberg theorem [1948], and of a theorem of Stein [1952].

THEOREM 14. Let $B_{1} \geqq 0, B_{1} \neq 0, r\left(B_{1}\right)<1$ and $B_{2} \geqq 0$, and assume that $B=$ $B_{1}+B_{2}$ is $K$-irreducible. Set $H=\left(I-B_{1}\right)^{-1} B_{2}$. Then one of the following three conditions holds:

(i) $r(H)<r(B)<1$,

(ii) $r(H)=r(B)=1$,

(iii) $r(H)>r(B)>1$.

Proof. Let $\mu=r(B)$. Then, by Theorem 6, $B x=\mu x$ for some $x>0$ and hence

$$
B_{2} x+(1-\mu) B_{1} x=\mu x-\mu B_{1} x .
$$

If $\mu=1$, this implies that $H x=x, x>0$, and hence, by Theorem 13 , that $r(H)=1$. In the case $\mu<1$, we have

$$
H x \leqq H x+(1-\mu)\left(I-B_{1}\right)^{-1} B_{1} x=\mu x, \quad x>0,
$$

where $H x \neq \mu x$ because of $B_{1} \neq 0$. Thus, Theorem 8 implies that $r(H)<\mu$. Finally, if $\mu>1$, then (4) gives

$$
H x \geqq H x-(\mu-1)\left(I-B_{1}\right)^{-1} B_{1} x=\mu x, \quad x>0,
$$

where once more $H x \neq \mu x$, and then by Theorem $10, r(H)>\mu$.

THEOREM 15. Let $A \in L(V)$ be symmetric and positive definite. If $B \in L(V)$ is such that $A-B^{T} A B$ is positive definite, then $r(B)<1$.

Proof. Let $V$ be the space of symmetric linear transformations on $R^{n}$, and let $K$ be the cone

$$
K=\{T \in V \mid T \text { is positive semidefinite }\} .
$$

The linear transformation $B$ on $V$ defined by

$$
B(T)=B^{T} T B
$$

is nonnegative, with respect to this cone, and the hypotheses state that

$$
B(A)<A \text { where } A>0 \text {. }
$$

Hence, by Theorem $3, r(B)<1$. Using the norm

$$
\|B\|=\sup _{\|T\|_{2}=1}\|B(T)\|_{2}=\|B\|_{2}^{2}
$$

where $\|\cdot\|_{2}$ is the spectral norm on $V$, we see that also $r(B)<1$.

Computer Science Center

University of Maryland

College Park, Maryland 20742 
1967. G. BIRKHOFF, "Linear transformations with invariant cones," Amer. Math. Monthly, v. 74, 1967, pp. 274-276. MR 35 \#5454.

1966. E. BoHL, "Eigenwertaufgaben bei monotonen Operatoren und Fehlerabschätzungen für Operatorgleichungen," Arch. Rational Mech. Anal., v. 22, 1966, pp. 313-332. MR 38 \#2622.

1958. K. FAN, "Topological proofs for certain theorems on matrices with non-negative elements," Monatsh. Math., v. 62, 1958, pp. 219-237. MR 20 \#2354.

1908. G. FrobeniUs, "Über Matrizen aus positiven Elementen," S.-B. Deutsch. Akad. Wiss. Berlin, v. 1908, pp. 471-476.

1909. G. FrobEnIUs, "Uber Matrizen aus positiven Elementen. II," S.-B. Deutsch. Akad. Wiss. Berlin, v. 1909, pp. 514-518.

1912. G. FrobeniUs, "Uber Matrizen aus nicht negativen Elementen," S.-B. Deutsch. Akad. Wiss. Berlin, v. 1912, pp. 456-477.

1964. A. Housenolder, The Theory of Matrices in Numerical Analysis, Blaisdell, Waltham, Mass., 1964. MR 30 \#5475.

1939. L. Kantorovič, "The method of successive approximations for functional equations," Acta Math., v. 71, 1939, pp. 63-97. MR 1, 18.

1950. M. G. KREIN \& M. A. RUTMAN, "Linear operators leaving invariant a cone in a Banach space," Uspehi Mat. Nauk, v. 3, 1948, no. 1 (23), pp. 3-95; English transl., Amer. Math. Soc. Transl. (1), v. 10, 1962, pp. 199-325. MR 10, 256; MR 12, 342.

1907. O. Perron, "Zur Theorie der Matrizen," Math. Ann., v. 64, 1907, pp. 248-263. 3.3 \# 1689.

1966. H. H. SchaEfER, Topological Vector Spaces, Macmillan, New York, 1966. MR

1972. H. Schneider \& R. E. L. Turner, "Positive eigenvectors of order-preserving maps," J. Math. Anal. Appl., v. 37, 1972, pp. 506-515.

1948. P. STEIN \& R. L. Rosenberg, "On the solution of linear simultaneous equations by iteration," J. London Math. Soc., v. 23, 1948, pp. 111-118. MR 10, 485.

1952. P. Stein, "Some general theorems on iterants," J. Res. Nat. Bur. Standards, v. 48, 1952 , pp. 82-83.

1968. J. S. VANDERGRAFT, "Spectral properties of matrics which have invariant cones," SIAM J. Appl. Math., v. 16, 1968, pp. 1208-1222. MR 39 \#599.

1972. J. S. VANDERGRAFT, "Applications of partial orderings to the study of positive definiteness, monotonicity, and convergence," SIAM J. Numer. Anal., v. 9, 1972, pp. 97-104. 1962. R. VARGA, Matrix Iterative Analysis, Prentice-Hall, Englewood Cliffs, N.J., 1962. MR 28 \#1725. 